8-1-1993

\title{
Diffraction of a Gaussian Beam by a Spherical Obstacle
}

James A. Lock

Cleveland State University, j.lock@csuohio.edu

Edward A. Hovenac

Sverdrup Technology Inc./NASA Lewis Research Center

Follow this and additional works at: https://engagedscholarship.csuohio.edu/sciphysics_facpub

Part of the Physics Commons

How does access to this work benefit you? Let us know!

\section{Publisher's Statement}

Copyright 1993 American Association of Physics Teachers. The article appeared in American Journal of Physics 61 (1993): 698-707 and may be found at http://aapt.scitation.org/doi/ 10.1119/1.17440

\section{Original Citation}

Lock, James A. and Edward A. Hovenac. "Diffraction of a Gaussian Beam by a Spherical Obstacle." American Journal of Physics 61 (1993): 698-707.

\section{Repository Citation}

Lock, James A. and Hovenac, Edward A., "Diffraction of a Gaussian Beam by a Spherical Obstacle" (1993). Physics Faculty Publications. 17.

https://engagedscholarship.csuohio.edu/sciphysics_facpub/17

This Article is brought to you for free and open access by the Physics Department at EngagedScholarship@CSU. It has been accepted for inclusion in Physics Faculty Publications by an authorized administrator of EngagedScholarship@CSU. For more information, please contact library.es@csuohio.edu. 


\title{
Diffraction of a Gaussian beam by a spherical obstacle
}

\author{
James A. Lock
}

Edward A. Hovenac

The Kirchhoff integral for diffraction in the near-forward direction is derived from the exact solution of the electromagnetic boundary value problem of a focused Gaussian laser beam incident on a spherical particle. The diffracted intensity in the vicinity of the particle is computed and the way in which the features of the diffraction pattern depend on the width of the Gaussian beam is commented on.

\section{INTRODUCTION}

For the situation of a wave incident on either a small aperture or obstacle, diffraction is loosely defined as the slight bending of the wave as it passes the edges of the aperture or obstacle. The most correct way to calculate the diffraction of light by a small aperture is to obtain the exact solution to the electromagnetic boundary value problem of a light wave incident on a flat plate that contains the aperture. The total electric field on the incident side of the plate is interpreted as the sum of the incoming wave and a reflected wave, while the electric field on the transmitted side is interpreted as the sum of the wave transmitted through the aperture plus the wave diffracted by it. ${ }^{\prime}$ Unfortunately, there are few aperture geometries for which an exact solution to the electromagnetic boundary value problem may be obtained, and the solutions are complicated. ${ }^{2}$

As an alternative to the exact approach, diffraction is almost always calculated using the Kirchhoff diffraction integral. ${ }^{3,4}$ Mathematically, the diffraction integral treats many aspects of the problem incorrectly. But, in spite of its mathematical shortcomings, its predictions agree closely with the results of experiments. ${ }^{5}$

For diffraction by a small obstacle, again exact solutions to the electromagnetic boundary value problem are known for a few simple geometries such as a disk, ${ }^{6}$ a sphere, ${ }^{7}$ an infinitely long strip, ${ }^{8}$ and an infinitely long cylinder. ${ }^{9}$ For these situations, the electric field on the transmitted side of the obstacle contains both the diffracted wave and waves that result from the interaction of the incident beam with the object's specific shape and composition. These interactions include specular reflection from the surface of the obstacle and transmission through it following an arbitrary number of internal reflections.

Again as an alternative to this exact approach, diffraction by an obstacle is almost always calculated using the Kirchhoff diffraction integral accompanied by Babinet's principle which states that the diffracted field of the obstacle is the incident field minus the diffracted field of its complementary aperture. ${ }^{10}$ The diffraction integral applied to an obstacle ignores its composition and considers only its projection onto an equivalent aperture plane. ${ }^{11}$ But again its predictions closely agree with the results of experiments. ${ }^{12-14}$

Recently, scattering of a Gaussian laser beam by a spherical particle has been solved exactly. ${ }^{15,16}$ While the solution has led to many interesting results concerning Gaussian beam scattering, it also allows for a deeper understanding of diffraction by obstacles. In laboratory situations, one often examines diffraction by a single obstacle. Thus one must arrange the experiment so that the weakly diffracted light at the detector is not overwhelmed by the intensity of the incident beam. This is accomplished in one of two ways. When employing a plane wave as the incident beam, one places a lens between the obstacle and the detector with the detector located in the focal plane of the lens. For this lens placement, the incident plane wave is focused to a point on the detector plane while the diffracted wave extends to either side. ${ }^{17}$ One may instead illuminate the obstacle by a narrow Gaussian beam from a laser and not employ a lens in front of the detector. For this situation, the diffraction pattern extends far to either side of the transverse width of the beam. Gaussian beam scattering theory describes this second experimental situation in a natural way.

In this paper, we examine two aspects of the diffraction of a focused Gaussian laser beam by a spherical obstacle in order to better understand diffraction as it applies to obstacles. First, employing the exact solution to the electromagnetic boundary value problem, we numerically generate a gray level plot of the diffracted intensity in the vicinity of the obstacle. The results visually confirm that diffraction is produced by an interaction of the incident beam with the edges of the obstacle. ${ }^{18-20}$ We then answer the question of why the Kirchhoff diffraction integral works as well as it does by deriving it from the exact solution to the spherical obstacle problem. Although other derivations of the Kirchhoff integral have been carried out before for plane-wave incidence, the Gaussian beam problem has a richness of physical interpretation not enjoyed by the plane-wave case.

Before examining these two aspects of diffraction by an obstacle, a certain amount of preparatory material must be presented. First the equations describing a focused Gaussian beam must be given and described. This is done in Sec. II with particular emphasis placed on the rate of spreading of the beam in the far zone. Similarly, the exact solution to the electromagnetic boundary value problem must be written down. This is done in Sec. III. As one might expect, the solution is rather complicated. But our analysis of diffraction does not require a detailed understanding of all the intricacies present in the exact solution. Next, in order to assess the effects of diffraction, it must be precisely defined. In order to define diffraction, however, we must also catalog all the other physical processes that are present in near-forward direction scattering. This is done in Sec. IV. After these three sections of preparatory material, in Sec. V we present a gray level plot of the diffracted intensity in the 


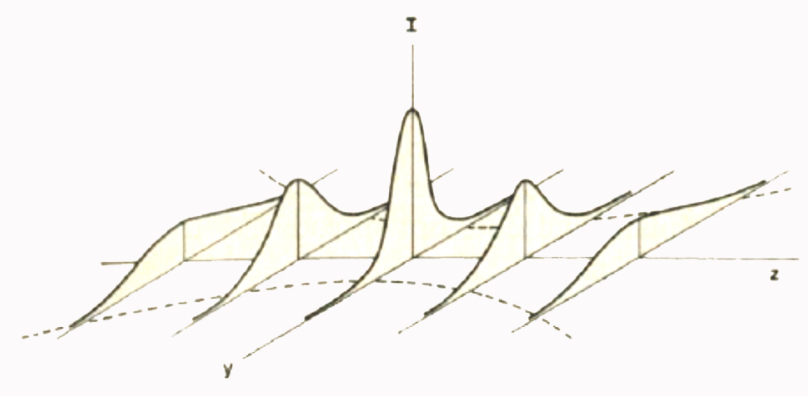

Fig. 1. Intensity of a Gaussian beam as a function of position in the $y z$ plane. The beam is propagating in the positive $z$ direction and the center of the focal waist is at $y=z=0$. The dashed lines represent the nominal width of the beam as a function of $z$.

vicinity of the spherical obstacle and physically interpret the various features of the plot. In Sec. VI we then derive the Kirchhoff diffraction integral from the exact solution of the scattering problem given in Sec. III and comment on the approximations under which the derivation is made. The diffraction integral is then used to examine the way in which the features of the diffraction pattern depend on the width of the incident Gaussian beam.

\section{THE INCIDENT GAUSSIAN BEAM}

Consider a laser beam propagating in the positive $z$ direction and linearly polarized in the $x$ direction. In the $z=0$ plane, the amplitude of the electric and magnetic fields is taken to have a Gaussian envelope of half-width $w$,

$$
\begin{aligned}
& \mathbf{E}_{\text {inc }}(x, y, 0, t)=E_{0} e^{-\left(x^{2}+y^{2}\right) / \omega^{2}} e^{-i \omega t} \hat{u}_{x}, \\
& \mathbf{B}_{\text {inc }}(x, y, 0, t)=\frac{E_{0}}{c} e^{-\left(x^{2}+y^{2}\right) / \omega^{2}} e^{-i \omega t} \hat{u}_{y} .
\end{aligned}
$$

Since this beam has some degree of confinement in the $z=0$ plane, it spreads as it propagates. This spreading is qualitatively illustrated in Fig. 1. As a first approximation to the propagation of the Gaussian beam to the point $(x, y, z)$, we employ the Fresnel diffraction formula,

$$
\begin{aligned}
\mathbf{U}(x, y, z, t)= & \frac{-i k}{2 \pi z} e^{i k z} \int_{-\infty}^{\infty} d x_{0} \int_{-\infty}^{\infty} d y_{0} \mathbf{U}\left(x_{0}, y_{0}, 0, t\right) \\
& \times e^{(i k / 2 z)\left(x-x_{0}\right)^{2}} e^{(i k / 2 z)\left(y-y_{0}\right)^{2}}
\end{aligned}
$$

with $\mathbf{U}=\mathbf{E}_{\text {inc }}$ or $\mathbf{B}_{\text {inc }}$ and

$$
k=\frac{2 \pi}{\lambda}=\frac{\omega}{c},
$$

to obtain

$$
\begin{aligned}
& \mathbf{E}_{\text {inc }}(x, y, z, t)=\frac{E_{0} e^{i k z}}{1+2 i z / k w^{2}} \exp \left[\frac{-\left(x^{2}+y^{2}\right) / w^{2}}{1+2 i z / k w^{2}}\right] e^{-i \omega t} \hat{u}_{x} . \\
& \mathbf{B}_{\text {inc }}(x, y, z, t)=\frac{E_{0} e^{i k z} / c}{1+2 i z / k w^{2}} \exp \left[\frac{-\left(x^{2}+y^{2}\right) / w^{2}}{1+2 i z / k w^{2}}\right] e^{-i \omega t} \hat{u}_{y} .
\end{aligned}
$$

Unfortunately Eq. (4) is not a solution to Maxwell's equations. An exact solution for a Gaussian beam may be obtained as an infinite series expansion in powers of ${ }^{21,22}$

$$
s=\frac{1}{k w} .
$$

For beams that are not especially tightly focused in the $z=0$ plane (i.e., $s \lesssim 0.01$ or $w \gtrsim 10 \mu \mathrm{m}$ when $\lambda=0.6328$ $\mu \mathrm{m})$, the so-called Davis first-order beam approximation which retains terms only of order $s$ in the expressions for the fields gives ${ }^{21}$

$$
\begin{aligned}
\mathbf{E}_{\text {inc }}(x, y, z, t)= & \frac{E_{0}}{D} e^{i(k z-\omega t)} \exp \left(\frac{-\left(x^{2}+y^{2}\right)}{w^{2} D}\right) \\
& \times\left(\hat{u}_{x}-\frac{2 i x s}{w D} \hat{u}_{z}\right) \\
\mathbf{B}_{\text {inc }}(x, y, z, t)= & \frac{E_{0}}{c D} e^{i(k z-\omega t)} \exp \left[\frac{-\left(x^{2}+y^{2}\right)}{w^{2} D}\right] \\
& \times\left(\hat{u}_{y}-\frac{2 i y s}{w D} \hat{u}_{z}\right),
\end{aligned}
$$

where

$$
D=1+\frac{2 i z s}{w} .
$$

This is a solution to both Maxwell's equations and the electromagnetic wave equation to about 3 parts in $10^{4}$. It differs from Eq. (4) only by the presence of the $z$ components of the fields. In Eq. (6), the focal plane of the beam is the $z=0$ plane, the center of the focal waist is at the origin of coordinates, and the length of the focal waist to either side of the focal plane is roughly $w / s$. The beam of Eq. (6) is called an on-axis beam since the beam envelope is symmetric about the $z$ axis.

The various terms in Eq. (6) have the following physical interpretations. The exponential of the real part of $-\left(x^{2}\right.$ $\left.+y^{2}\right) / w^{2} D$ represents the Gaussian falloff of the electric and magnetic field envelopes. The factors $e^{i k z}$ and the exponential of the imaginary part of $-\left(x^{2}+y^{2}\right) / w^{2} D$ represent an approximation to the curvature of the planes of constant phase to either side of the focal plane. The magnitude of the $1 / D$ factor in the second exponential describes the increased width of the Gaussian beam to either side of the focal plane, and the overall $1 / D$ factor describes the decreased amplitude that accompanies the spreading of the beam as required by conservation of energy. The $w \rightarrow \infty$ limit of the Gaussian beam of Eq. (6) is

$$
\begin{aligned}
& \mathbf{E}_{\text {inc }}(x, y, z, t)=E_{0} e^{i(k z-\omega t)} \hat{u}_{x}, \\
& \mathbf{B}_{\text {inc }}(x, y, z, t)=\frac{E_{0}}{c} e^{i(k z-\omega t)} \hat{u}_{y},
\end{aligned}
$$

which describes a plane wave. When a Gaussian beam emerges from a laser, it is usually diverging slightly and a virtual focal plane exists far behind the laser. If a laser beam is focused by a lens, however, the focal plane of the beam becomes the focal plane of the lens.

In the near zone of the beam defined as those values of $z$ for which the beam has begun to spread beyond the size of its focal waist, we have $z>k w^{2} / 2$ and the factors of $D$ in Eq. (6) may be Taylor series expanded in powers of $k w^{2} /$ $2 i z$ to give 


$$
\begin{aligned}
\mathbf{E}_{\text {inc }}(x, y, z, t)= & \frac{-i E_{0}(k w)^{2}}{2 k z} e^{i(k z-\omega t)} \exp \left[\frac{i k\left(x^{2}+y^{2}\right)}{2 z}\right. \\
& \left.\times\left(1-\frac{i k w^{2}}{2 z}\right)^{-1}\right]\left(\hat{u}_{x}-\frac{x}{z} \hat{u}_{z}\right), \\
\mathbf{B}_{\text {inc }}(x, y, z, t)= & \frac{-i E_{0}(k w)^{2}}{2 c k z} e^{i(k z-\omega t)} \exp \left[\frac{i k\left(x^{2}+y^{2}\right)}{2 z}\right. \\
& \left.\times\left(1-\frac{i k w^{2}}{2 z}\right)^{-1}\right]\left(\hat{u}_{y}-\frac{y}{z} \hat{u}_{z}\right) .
\end{aligned}
$$

In the near-forward direction defined by $\left(x^{2}+y^{2}\right)^{1 / 2}<z$ and in the far zone or Fraunhofer zone of the beam $z \gg \theta^{2} k^{3} w^{4}$ where only the first two terms in the Taylor series expansion of $\left[1-\left(i k w^{2} / 2 z\right)\right]^{-1}$ in the exponential are retained in analogy to Fraunhofer diffraction by an aperture, the equation of the beam becomes

$\mathbf{E}_{\text {inc }}(\mathbf{r}, t)=\frac{-i E_{0}(k w)^{2}}{2 k r} e^{i(k r-\omega t)} e^{-(k \omega \theta / 2)^{2}}\left(\hat{u}_{x}-\theta \cos \phi \hat{u}_{z}\right)$,

$\mathbf{B}_{\text {inc }}(\mathbf{r}, t)=\frac{-i E_{0}(k w)^{2}}{2 c k r} e^{i(k r-\omega t)} e^{-(k w \theta / 2)^{2}}\left(\hat{u}_{y}-\theta \sin \phi \hat{u}_{z}\right)$,

where

$$
r=\left(x^{2}+y^{2}+z^{2}\right)^{1 / 2}
$$

and

$$
\sin \theta \approx \theta \approx \frac{\left(x^{2}+y^{2}\right)^{1 / 2}}{z} .
$$

Since the beam focal waist is confined to a small region about the origin and thereby resembles a point source, the beam propagates in the far zone as an outgoing spherical wave centered at the origin. The size of its focal waist and its far-zone angular spreading satisfy an uncertainty relation. If the beam is tightly confined at the focal waist, it spreads quickly as it propagates and has substantial angular spreading in the far zone. Conversely, if the beam is weakly confined at the focal waist, it spreads slowly as it propagates and has only minor angular spreading in the far zone. In the limit $w \rightarrow \infty$, a plane wave has an infinite width in the focal plane and does not spread.

\section{THE SCATTERED WAVE}

In this section, we briefly summarize the formulas for scattering of a Gaussian laser beam by a spherical particle. These formulas are not presented as an end in themselves, but only as a reference point from which to elucidate the physical meaning of the diffraction process. Those readers not interested in the mathematical details of Gaussian beam scattering may immediately proceed to Secs. IV and V and to Sec. VI after Eq. (42) where the physical implications of our results are discussed.

Consider the on-axis focused Gaussian beam of Eq. (6) incident on a spherical particle of radius $a$ and refractive index $n$ whose center is at the origin of coordinates. Exterior to the particle, the fields have the form

$$
\begin{aligned}
& \mathbf{E}_{\text {total }}(\mathbf{r})=\mathbf{E}_{\text {inc }}(\mathbf{r})+\mathbf{E}_{\text {scattered }}(\mathbf{r}), \\
& \mathbf{B}_{\text {total }}(\mathbf{r})=\mathbf{B}_{\text {inc }}(\mathbf{r})+\mathbf{B}_{\text {scattered }}(\mathbf{r}) .
\end{aligned}
$$

For future use, we will assume that the radial component of the incident electric field may be written as

$$
\mathbf{E}_{\text {inc }}(\mathbf{r}) \cdot \hat{u}_{r}=E_{0} e^{i k r \cos \theta} f(k r, \theta) \sin \theta \cos \phi,
$$

in spherical coordinates. The incident field for a focused on-axis Davis first-order Gaussian beam has this form with

$$
f(k r, \theta)=\frac{1}{D}\left(1-\frac{2 i s r \cos \theta}{w D}\right) \exp \left(\frac{-r^{2} \sin ^{2} \theta}{w^{2} D}\right),
$$

and a plane wave has $f(k r, \theta)=1$.

At an arbitrary distance $r \geqslant a$ from the center of the spherical particle, the scattered fields of Eq. (13) are written as an infinite series of partial waves ${ }^{15,16}$

$$
\begin{aligned}
\mathbf{E}_{\text {scattered }}(\mathbf{r})= & \frac{i E_{0} \sin \theta \cos \phi}{k r} \hat{u}_{r} \sum_{l=1}^{\infty} i^{l}(2 l+1) I_{l} a_{l} h_{l}^{(1)}(k r) \pi_{l}(\theta)-E_{0} \cos \phi \hat{u}_{\theta} \sum_{l=1}^{\infty} i^{l} \frac{2 l+1}{l(l+1)}\left\{I b_{l} h_{l}^{(1)}(k r) \pi_{l}(\theta)\right. \\
& \left.-i I a_{l}\left[h_{l-1}^{(1)}(k r)-\frac{l}{k r} h_{l}^{(1)}(k r)\right] \tau_{l}(\theta)\right\}+E_{0} \sin \phi \hat{u}_{\phi} \sum_{l=1}^{\infty} i^{l} \frac{2 l+1}{l(l+1)}\left\{I_{l} b_{l} h_{l}^{(1)}(k r) \tau_{l}(\theta)-i I a_{l}\left[h_{l-1}^{(1)}(k r)\right.\right. \\
& \left.\left.-\frac{l}{k r} h_{l}^{(1)}(k r)\right] \pi_{l}(\theta)\right\} \\
\mathbf{B}_{\text {scattered }}(\mathbf{r})= & \frac{i E_{0} \sin \theta \sin \phi}{c k r} \hat{u}_{r} \sum_{l=1}^{\infty} i^{l}(2 l+1) I b_{l} h_{l}^{(1)}(k r) \pi_{l}(\theta)-\frac{E_{0} \sin \phi}{c} \hat{u}_{\theta} \sum_{l=1}^{\infty} i^{l} \frac{2 l+1}{l(l+1)}\left\{I a_{l} h_{l}^{(1)}(k r) \pi_{l}(\theta)\right. \\
& \left.-i I b_{l}\left[h_{l-1}^{(1)}(k r)-\frac{l}{k r} h_{l}^{(1)}(k r)\right] \tau_{l}(\theta)\right\}-\frac{E_{0} \cos \phi}{c} \hat{u}_{\phi} \sum_{l=1}^{\infty} i^{l} \frac{2 l+1}{l(l+1)}\left\{I a_{l} h_{l}^{(1)}(k r) \tau_{l}(\theta)-i I b_{l}\left[h_{l-1}^{(1)}(k r)\right.\right. \\
& \left.\left.-\frac{l}{k r} h_{l}^{(1)}(k r)\right] \pi_{l}(\theta)\right\} .
\end{aligned}
$$

In these expressions the partial wave scattering amplitudes are given by

$$
a_{l}=\frac{j_{l}(k a) j_{l-1}(n k a)-n j_{l-1}(k a) j_{l}(n k a)-\left(\left[\left(n^{2}-1\right) / n\right]\right)(l / k a) j_{l}(k a) j_{l}(n k a)}{h_{l}^{(1)}(k a) j_{l-1}(n k a)-n h_{l-1}^{(1)}(k a) j_{l}(n k a)-\left(\left[\left(n^{2}-1\right) / n\right]\right)(l / k a) h_{l}^{(1)}(k a) j_{l}(n k a)},
$$




$$
\begin{aligned}
b_{l}= & \frac{n j_{l}(k a) j_{l-1}(n k a)-j_{l-1}(k a) j_{l}(n k a)}{n h_{l}^{(1)}(k a) j_{l-1}(n k a)-h_{l-1}^{(I)}(k a) j_{l}(n k a)}, \\
I_{l}= & \frac{(-i)^{l-1} k a}{2 l(l+1) j_{l}(k a)} \\
& \times \int_{0}^{\pi} \sin ^{2} \theta d \theta f(k a, \theta) e^{i k a \cos \theta} P_{l}^{1}(\cos \theta),
\end{aligned}
$$

where $j_{l}$ and $n_{l}$ are spherical Bessel functions and spherical Neumann functions, and $h_{l}^{(1)}$ is the spherical Hankel function of the first kind

$$
h_{l}^{(1)}=j_{l}+i n_{l} \text {. }
$$

The factors $a_{l}$ and $b_{l}$ are the partial wave scattering amplitudes for an incident plane wave and $I_{l}$ describes the influence of the shape of the incident beam on the scattering. The dependence on the scattering angle $\theta$ is given by the angular functions

$$
\begin{aligned}
& \pi_{l}(\theta)=\frac{1}{\sin \theta} P_{l}^{l}(\cos \theta), \\
& \tau_{l}(\theta)=\frac{d}{d \theta} P_{l}^{1}(\cos \theta),
\end{aligned}
$$

where $P_{l}^{1}$ are associated Legendre polynomials.

It is tempting to assume that all the effects of scattering in Eq. (13) are contained solely in $\mathbf{E}_{\text {scattered }}$ and $\mathbf{B}_{\text {scattered }}$. This is often but not always the case. When the incident wave and the scattered wave spatially overlap, the effects of scattering are also contained in the interference between the two waves rather than in the scattered wave alone. This overlap occurs in the near zone regardless of whether the incident field is a plane wave ${ }^{23}$ or a Gaussian beam, ${ }^{16}$ and in the far zone for plane-wave incidence only at $\theta=0^{\circ}$. For Gaussian beam incidence substantial overlap also occurs in the far zone for the larger angular region $\theta \lesssim 1 / k w$ due to the spreading of the incident beam. ${ }^{24}$ We will see in Sec. 6 that interference of the incident beam and scattered beam in the region of spatial overlap has a major effect on the observed diffraction pattern.

In the far zone of the scattered wave where $k r \gg 1, \mathrm{Eq}$. (16) assumes the somewhat simpler form,

$\mathbf{E}_{\text {scattered }}(\mathbf{r})=\frac{-i E_{0}}{k r} e^{i k r}\left[-S_{2}(\theta) \cos \phi \hat{u}_{\theta}+S_{1}(\theta) \sin \phi \hat{u}_{\phi}\right]$,

$\mathbf{B}_{\text {scattered }}(\mathbf{r})=\frac{-i E_{0}}{c k r} e^{i k r}\left[-S_{1}(\theta) \sin \phi \hat{u}_{\theta}-S_{2}(\theta) \cos \phi \hat{u}_{\phi}\right]$,

with

$$
\begin{aligned}
& S_{1}(\theta)=\sum_{l=1}^{\infty} \frac{2 l+1}{l(l+1)} I_{l}\left[a_{l} \pi_{l}(\theta)+b_{l} \tau_{l}(\theta)\right], \\
& S_{2}(\theta)=\sum_{l=1}^{\infty} \frac{2 l+1}{l(l+1)} I_{l}\left[a_{l} \tau_{l}(\theta)+b_{l} \pi_{l}(\theta)\right],
\end{aligned}
$$

where we have used

$$
\lim _{k r>1} h_{l}^{(1)}(k r)=\frac{(-i)^{l+1}}{k r} e^{i k r}
$$

The infinite series of partial waves $l$ of Eqs. (16) and (24) is initially very slowly convergent, and then rapidly converges when ${ }^{25}$

$$
l_{\max } \approx k a+4(k a)^{1 / 3}+2 .
$$

Thus the infinite series is standardly truncated at $l_{\max }$ when doing numerical computations. For plane-wave incidence with $I_{l}=1$, Eqs. (13), (17)-(18), (21)-(24), and (26) are known as the Mie solution for plane-wave scattering by a sphere. ${ }^{7}$

\section{THE DIFFRACTED PORTION OF THE SCATTERED WAVE}

The greatest difficulty in employing Eq. (16) when considering diffraction by a spherical obstacle is that the equation gives the entire scattered wave, not just the contribution of diffraction. One must carefully define diffraction and then separate it from all the other physical processes that contribute to scattering in the near-forward direction. This definition and separation are facilitated by decomposing the partial wave scattering amplitudes $a_{l}$ and $b_{l}$ of Eqs. (17) and (18) into a series of successive interactions of the incident partial waves with the surface of the spherical obstacle. A similar decomposition into a series of multiple internal reflections is often made for a plane wave incident on a thin film with flat surfaces. For each partial wave for the spherical obstacle problem we have

$$
\left.\begin{array}{l}
a_{l} \\
b_{l}
\end{array}\right]=\frac{1}{2}\left[1-R_{l}^{22}-\sum_{p=1}^{\infty} T_{l}^{21}\left(R_{l}^{11}\right)^{p-1} T_{l}^{12}\right] .
$$

The explicit forms for $R_{l}^{22}, T_{l}^{21}, R_{l}^{11}$, and $T_{l}^{12}$ in terms of spherical Bessel functions and spherical Neumann functions are given elsewhere. ${ }^{26,27}$

Equation (27) is called the Debye series. It is useful in understanding the scattering process since each term in the series has a straightforward physical interpretation. The term $R_{l}^{22}$ is the fraction of the incident partial wave amplitude that is reflected from the surface of the spherical particle, and $T_{l}^{21}\left(R_{l}^{11}\right)^{p-1} T_{l}^{12}$ is the fraction of the amplitude that is transmitted into the sphere $\left(T_{l}^{21}\right)$ and is internally reflected $p-1$ times $\left(R_{!}^{11}\right)^{p-1}$ before being transmitted outward $\left(T_{l}^{12}\right){ }^{28}$ These terms depend on both the size and refractive index of the spherical particle. When they are inserted for $a_{l}$ and $b_{l}$ into Eq. (24) and the sums over partial waves are performed for $l \leqslant k a$ in the short wavelength limit $k a>1$, the largest contribution to the result is interpreted as the electric field produced by the family of geometrical light rays that are reflected by the sphere or transmitted through it after $p-1$ internal reflections respectively. ${ }^{26}$ The angle of incidence of an individual geometrical ray in this interpretation is given $b^{29}$

$$
\sin \theta_{i} \approx \frac{l}{k a},
$$

and is shown in Fig. 2(a). A smaller contribution to the specular reflection and transmission-internal reflection sums for $k a>1$ is provided by the radiation shed by electromagnetic surface waves ${ }^{26}$ that are created by the incoming light rays at grazing incidence with $\theta_{i} \approx 90^{\circ}$. These surface waves are illustrated in Fig. 2(b). 


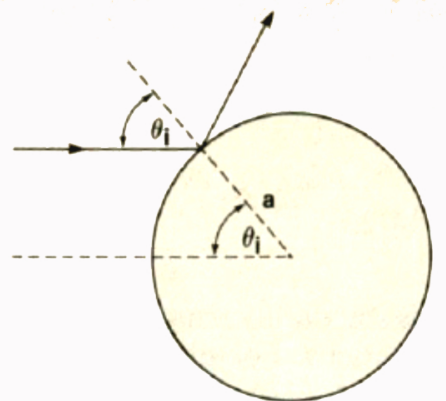

a

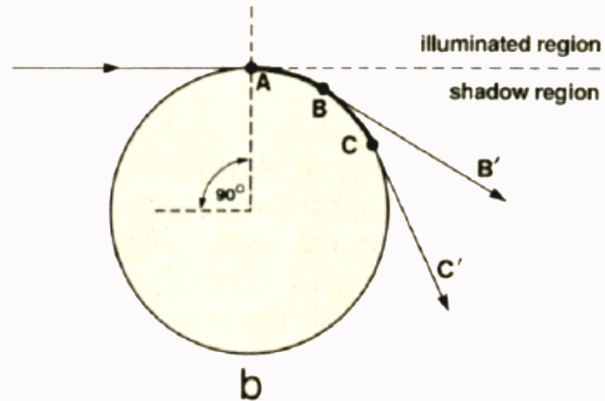

Fig. 2. (a) A specularly reflected geometrical light ray. The incoming ray is incident on the sphere with the angle $\theta_{i}$ (b) A light ray at grazing incidence $\left(\theta_{i}=90^{\circ}\right)$ at point $\mathrm{A}$ creates an electromagnetic surface wave that propagates along the perimeter to points $\mathrm{B}, \mathrm{C}$, and beyond. The surface wave sheds the electromagnetic waves $\mathrm{BB}^{\prime}$ and $\mathrm{CC}^{\prime}$ tangentially from the surface into the shadow region.

The partial waves $l>k a$ qualitatively correspond via Eq. (28) to light rays that miss striking the sphere. An interesting interpretation of their behavior results from examining the radial part of the wave equation for an individual partial wave. It resembles a Schrodinger-type equation with two potentials, a well potential describing the particle interior and a centrifugal potential surrounding it. ${ }^{30}$ In this interpretation, the $l>k a$ partial waves do not have enough energy to pass over the centrifugal potential and interact directly with the spherical particle. In order to interact with the particle at all, they must tunnel through the centrifugal barrier that surrounds it. This is illustrated in Fig. 3 (a). In this tunneling regime it has been shown that the amplitude for a partial wave to tunnel through the centrifugal barrier and then be transmitted into the particle is very small, i.e., ${ }^{31,32}$

$$
\lim _{l>k a} T_{l}^{21} \approx \lim _{l>k a} T_{l}^{12} \approx \exp \left(-\frac{1}{3} \frac{(l-k a)^{3 / 2}}{(k a)^{1 / 2}}\right) .
$$

But once inside the particle, the internal reflection amplitude for the partial wave is high, i.e.,

$$
\lim _{l>k a} R_{l}^{11} \approx 1 \text {. }
$$

Thus for the partial waves $l>k a$, the term $T_{l}^{21}\left(R_{l}^{11}\right)^{p-1} T_{l}^{12}$ continues to have a straightforward physical interpretation. It corresponds to the fraction of the incident partial wave that tunnels through the centrifugal barrier, is transmitted into the particle and internally reflects $p-1$ times, then leaves the particle and tunnels back out through the centrifugal barrier as is illustrated in Fig. 3(b).

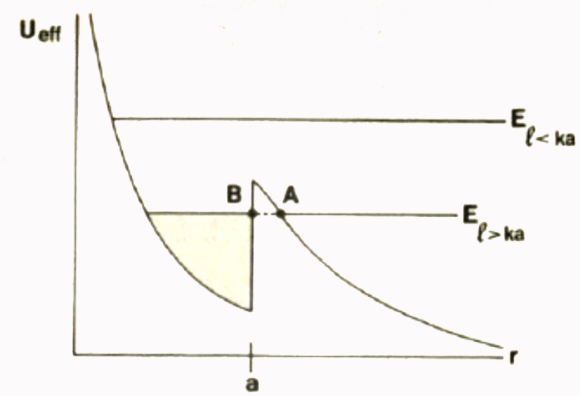

a
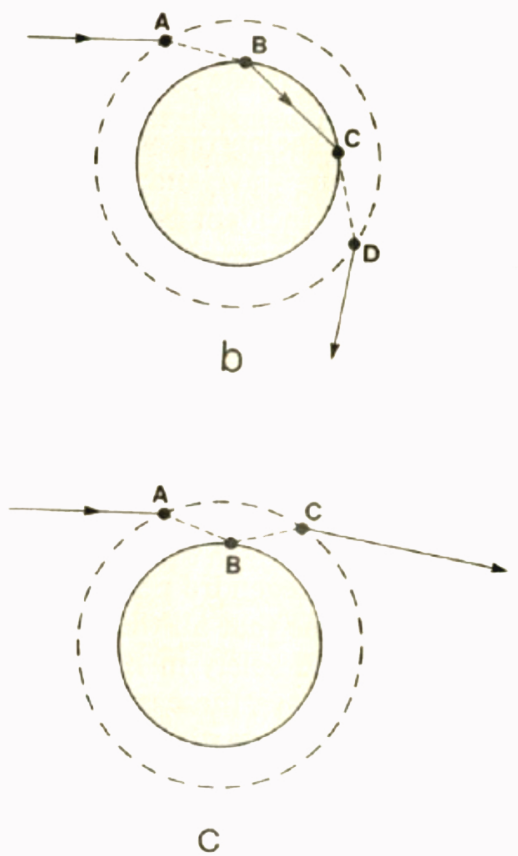

Fig. 3. (a) The effective potential of the radial wave equation for a spherical particle of radius $a$ as a function of $r$. For $l<k a$, the radial wave interacts directly with the particle. For $l>k a$, the radial wave must tunnel through the centrifugal potential that surrounds the particle from A to B in order to interact with it. (b) The process of tunneling transmission. The partial wave tunnels through the centrifugal potential (dashed curve) from A to B. It is transmitted into the particle (solid curve) at B and propagates to $\mathrm{C}$, where it tunnels back out through the centrifugal potential to D. (c) The process of tunneling reflection. The partial wave tunnels through the centrifugal potential (dashed curve) from A to B. It reflects off the particle surface (solid curve) at B, and tunnels back out through the centrifugal potential to $\mathrm{C}$.

The first term of Eq. (27) is independent of the refractive index of the particle. Its contribution to scattering is identical for a dielectric sphere such as a water droplet, for a metallic sphere such as a ball bearing, or for a highly absorbing sphere such as a soot particle. Since this term is the only portion of Eq. (27) that is independent of the composition of the particle, the prescription

$$
\left.\begin{array}{l}
a_{l} \\
b_{l}
\end{array}\right\}=\frac{1}{2}
$$

may be considered as a definition of diffraction by an obstacle, i.e., diffraction is the portion of the scattering amplitude that is independent of the composition of the obstacle. ${ }^{33}$ The major difficulty with this definition is that Eq. (31) substituted into Eqs. (16) and (24) diverges when 
the infinite series over partial waves is performed. This difficulty may be overcome by noticing that the partial wave sum for specular reflection alone,

$$
\left.\begin{array}{l}
a_{l} \\
b_{l}
\end{array}\right\}=-\frac{1}{2} R_{l}^{22}
$$

also diverges since the specular reflection amplitude for large impact parameter partial waves is ${ }^{31}$

$$
\lim _{l>k a} R_{l}^{22} \approx 1-\exp \left(\frac{-2}{3} \frac{(l-k a)^{3 / 2}}{(k a)^{1 / 2}}\right) .
$$

When Eqs. (31)-(32) are combined as ${ }^{34}$

$$
\begin{aligned}
& a_{l} \\
& b_{l}
\end{aligned} \mid=\frac{1}{2}\left(1-R_{l}^{22}\right)
$$

the individual divergences cancel and the sum over partial waves rapidly converges at $l_{\max }$

For partial waves with $l \lesssim k a$, it is sensible to think of diffraction and specular reflection as distinct physical processes since diffraction contributes primarily to nearforward scattering while geometrical light rays incident via Eq. (28) are primarily reflected away from the forward direction. But $l \approx k a$ corresponds to incoming light rays at grazing incidence that are specularly reflected in the nearforward direction. From Eqs. (31) and (33), this contribution is almost exactly canceled by the diffraction contribution of the $l \approx k a$ partial waves. Thus while the terms 1 and $R_{l}^{22}$ may be thought of as two separate processes for $l \lesssim k a$, for partial waves $l \gtrsim k a$ their identities merge and it is more physically meaningful to consider $1-R_{l}^{22}$ as a single physical process. Since the second term in Eq. (33) is the amplitude for tunneling into and out of the centrifugal barrier, the process $1-R_{l}^{22}$ for partial waves with $l>k a$ may be given the name tunneling reflection, ${ }^{35-38}$ i.e., the partial waves tunnel through the centrifugal barrier, reflect off the particle surface, and then tunnel back out. This is illustrated in Fig. 3(c). Because of the rapid damping of the tunneling amplitude as $l$ is increased, tunneling reflection is much weaker than diffraction in the near-forward direction. Its major effects are to reduce the intensity of the first few diffraction secondary maxima and to cause the intensity at the first few diffraction minima to be nonzero. At larger scattering angles, tunneling reflection and diffraction become comparable but are each much weaker than the specular reflection of geometrical light rays. ${ }^{38}$

Thus the definition of diffraction may be revised, i.e., diffraction is the portion of the scattering amplitude for the partial waves $l \leqslant k a$ that is independent of the composition of the obstacle, since the portion for $l>k a$ is canceled by the specular reflection term of the Debye series. Even this revised definition leads to a number of surprising results. For example, Eq. (31) with $l \leqslant k a$ allows one to compute diffraction at all scattering angles, even in the backscattering hemisphere. This is curious since diffraction by the complementary aperture is limited by the presence of the aperture plane to $\theta<90^{\circ}$. However, for large angle diffraction where $\theta>1 / k a$, the diffracted electric field is proportional to $a^{1 / 2}$, and is dominated by the electric field due to scattered geometrical rays which is proportional to $a$. Thus for large spherical obstacles, large angle diffraction is negligible in comparison to geometrical ray scattering.
With the revised definition of diffraction, the physical picture of scattering given by Eq. (27) for $k a>1$ is that of diffraction plus specular reflection plus transmission following $p-1$ internal reflections for partial waves with $l \leqslant k a$, and tunneling reflection plus tunneling transmission following $p-1$ internal reflections for partial waves with $l>k a$. For small values of $k a$, the mathematical decomposition of Eq. (27) is still valid. But the individual terms of the Debye series do not have well-defined physical interpretations.

There are two ways in which to compute diffraction using the Debye series analysis of the partial wave scattering amplitudes. First, one may calculate diffraction as we have defined it in Eq. (31) and truncate the partial wave sum at $l=k a$. For this choice, the contributions to near-forward scattering provided by specularly reflected rays, surface waves, and by tunneling reflection are not considered. Second, one may calculate the combined effects of diffraction, specularly reflected rays, surface waves, and tunneling reflection using Eq. (34) and truncate the partial wave sum at $l_{\max }$. In producing the gray level plot of the diffracted intensity we use the second method, while in our derivation of the Kirchhoff diffraction integral we use the first method.

\section{DIFFRACTION IN THE VICINITY OF A SPHERICAL OBSTACLE}

We substituted Eq. (34) into Eq. (16) and numerically computed $\left|\mathbf{E}_{\text {total }}(\mathbf{r})\right|^{2}$ as in $\mathbf{E q}$. (13). The results are shown in the gray level plot of Fig. 4(a) for a focused Gaussian beam of half-width $w=10 \mu \mathrm{m}$ and $\lambda=0.6328 \mu \mathrm{m}$ incident on a spherical particle of radius $a=10 \mu \mathrm{m}$ and $n=1.333$. The center of the particle is at the center of the focal waist of the beam. The width of the Gaussian beam employed here is at the lower end of the region of applicability of the Davis first-order beam approximation.

The Gaussian beam in Fig. 4(a) is incident from the left. Black corresponds to the most intensely scattered light and white corresponds to the least intense scattering. The gray level plot of Fig. 4(a) verifies the scattering predictions of Ref. 39 which are illustrated in Fig. 4(b). The so-called deep shadow region where the particle has blocked the incident beam is to the right of the particle. Since the fullwidth of the beam is equal to the diameter of the particle, the Gaussian beam is substantially weaker in the regions where diffraction is important (i.e., above and below the particle to its right) than it is at the edges of the particle. Thus the diffracted intensity is not obscured in Fig. 4(a) by the incident beam as would have been the case if the particle had been illuminated by a plane wave or by a Gaussian beam with $w \gg a$. The interference pattern at the edge of the incident beam is the interference of the specularly reflected light rays with the incident beam. The portion of the incident beam that grazes the edge of the sphere creates electromagnetic surface waves that shed radiation tangentially as they propagate around the particle circumference. The interference pattern in the deep shadow region a few radii to the right of the sphere is due to the interference of two sprays of surface wave radiation that leave the sphere surface above and below the centerline respectively. The large intensity region along the centerline in the deep shadow region is due to axial focusing of all the sprays of surface wave radiation since they travel equal path lengths 


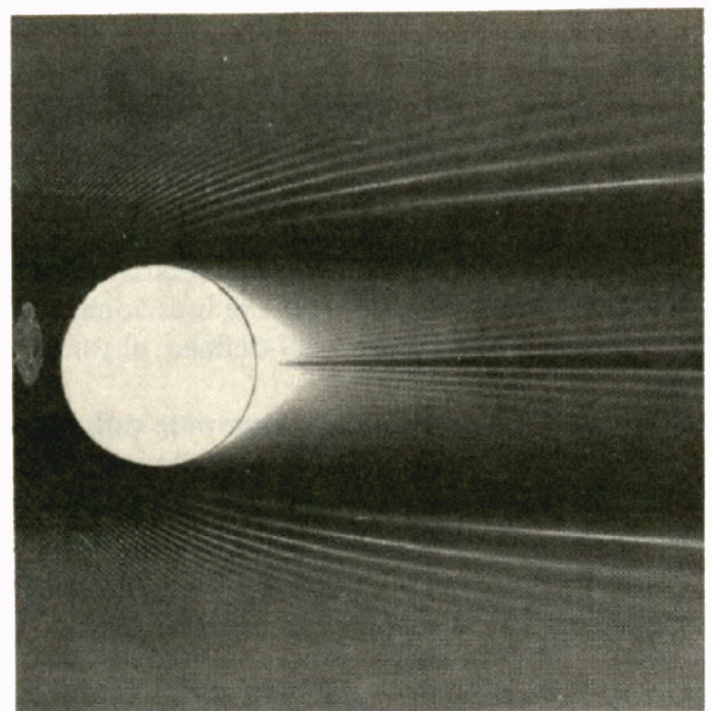

(a)

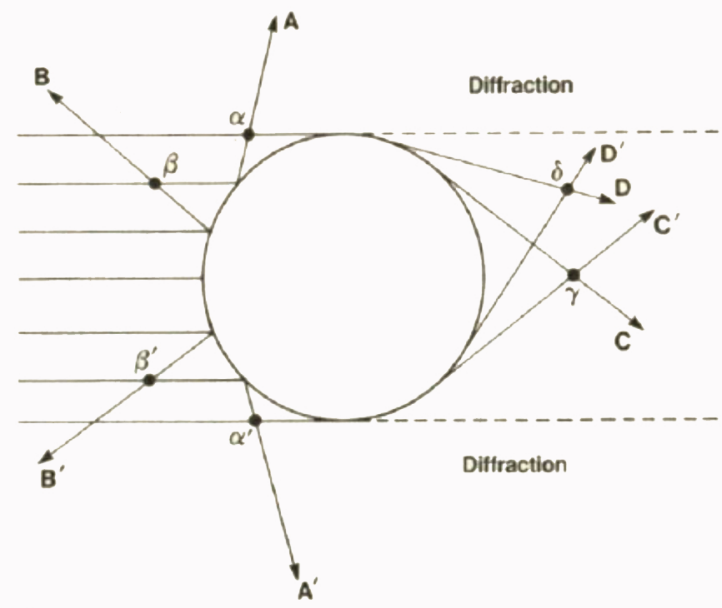

(b)

Fig. 4. (a) Intensity for diffraction, specularly reflected rays, surface waves, and tunneling reflection for a Gaussian beam of $\lambda=0.6328 \mu \mathrm{m}$ and $w=10.0 \mu \mathrm{m}$ incident on a spherical water droplet of $n=1.333$ and $a=10.0 \mu \mathrm{m}$. Black corresponds to the most intensely scattered light and white corresponds to the least intense scattering. The gray level scale is proportional to the logarithm of the intensity. (b) The specularly reflected rays $A, A^{\prime}, B$, and $B^{\prime}$ interfere with the incident Gaussian beam at $\alpha, \alpha^{\prime}, \beta$, and $\beta^{\prime}$. The deep shadow region is between the dashed lines to the right of the particle. The surface waves $\mathrm{D}$ and $\mathrm{D}^{\prime}$ interfere at $\delta$. The axial focusing of the surface waves $\mathbf{C}$ and $\mathbf{C}^{\prime}$ occurs at $\gamma$.

from their exit points on the sphere surface to the centerline. Lastly, a prominent broad interference pattern due to diffraction and resembling a Fresnel straight-edge pattern emanates from the upper and lower edges of the sphere. These two Fresnel-like patterns spread as they propagate to the right of the sphere, and eventually overlap to form the far-zone Fraunhofer diffraction pattern of the obstacle.

There is much mathematical and physical evidence that diffraction by either an obstacle or an aperture is dominated by the interaction of the incident light with the edges of the obstacle or aperture. ${ }^{18-20}$ The large scale Fresnel-like patterns emanating from the edges of the sphere in Fig. 4 (a) support the edge interaction interpretation of diffraction.

\section{DERIVATION OF THE KIRCHHOFF DIFFRACTION INTEGRAL}

As complicated as Eqs. (13)-(22) for Gaussian beam scattering may appear, they immediately simplify to manageable expressions if one is interested in diffraction as defined by Eq. (31) in the near-forward direction by a sphere much larger than the wavelength of the incident light. In the near-forward direction given by $\theta<1$ radian, the angular functions $\pi_{l}(\theta)$ and $\tau_{l}(\theta)$ of Eqs. (21) and $(22)$ have been shown to reduce to ${ }^{33}$

$$
\begin{aligned}
& \pi_{l}(\theta)=\frac{l(l+1)}{2}\left[J_{0}\left(\left(l+\frac{1}{2}\right) \theta\right)+J_{2}\left(\left(l+\frac{1}{2}\right) \theta\right)\right], \\
& \tau_{l}(\theta)=\frac{l(l+1)}{2}\left[J_{0}\left(\left(l+\frac{1}{2}\right) \theta\right)-J_{2}\left(\left(l+\frac{1}{2}\right) \theta\right)\right],
\end{aligned}
$$

where $J_{0}$ and $J_{2}$ are Bessel functions. Similarly, in the short wavelength limit where $k a \gg 1$, a stationary phase evaluation of the integral in Eq. (19) gives ${ }^{28,40,41}$

$$
I_{l}=\exp \left[\frac{-\left(l+\frac{1}{2}\right)^{2}}{(k w)^{2}}\right]
$$

The spherical Hankel function of Eq. (20) may be written as the infinite series ${ }^{42}$

$$
h_{l}^{(1)}(k r)=(-i)^{l+1} \frac{e^{i k r}}{k r} \sum_{j=0}^{l} \frac{i^{j}}{j !(2 k r)^{j}} \frac{(l+j) !}{(l-j) !} .
$$

In the limits $r>\lambda, r^{2} \gg a^{2}, r^{3} \gg a^{4} / \lambda$, etc., and using $(l+j) ! /$ $(l-j) ! \approx(l+1 / 2)^{2 j}$, each of the terms of the infinite series in Eq. (37) are well approximated by the corresponding terms of the Taylor series expansion of $\exp \left[i(l+1 / 2)^{2}\right.$, $2 k r]$. As a result,

$$
h_{l}^{(1)}(k r) \approx(-i)^{l+1} \frac{e^{i k r}}{k r} \exp \left[\frac{i\left(l+\frac{1}{2}\right)^{2}}{2 k r}\right]
$$

and

$$
\begin{aligned}
h_{l-1}^{(1)}(k r)-\frac{l}{k r} h_{l}^{(1)}(k r) \approx & (-i)^{l} \frac{e^{i k r}}{k r} \exp \left[\frac{i\left(l+\frac{1}{2}\right)^{2}}{2 k r}\right] \\
& \times\left(e^{-i l / k r}+\frac{i l}{k r}\right) .
\end{aligned}
$$

For $r^{2} \gg a^{2}$, the last exponential in Eq. (39) may be Taylor series expanded to cancel the il/ $k r$ factor. Our revised definition of diffraction in Eq. (31) requires that the sum over partial waves in Eq. (16) be truncated at $l=k a$. Finally combining Eqs. (13), (16), (31), (35), (36), (38), and (39), we obtain

$$
\begin{aligned}
\mathbf{E}_{\text {total }}^{\text {diffracted }}(\mathbf{r})= & \mathbf{E}_{\text {inc }}(\mathbf{r})+\frac{i E_{0}}{k r} e^{i k r}\left(\hat{u}_{x}-\theta \cos \phi \hat{u}_{z}\right) \\
& \times \sum_{l=1}^{k a}\left(l+\frac{1}{2}\right) J_{0}\left(\left(l+\frac{1}{2}\right) \theta\right) e^{i\left(l+\frac{1}{2}\right)^{2} / 2 k r} \\
& \times e^{-\left(l+\frac{1}{2}\right)^{2} /(k w)^{2}} .
\end{aligned}
$$


Since we assumed that $k a \gg 1$, the sum over partial waves in Eq. (40) may be approximated by an integral over an associated impact parameter giving

$$
\begin{aligned}
\mathbf{E}_{\text {total }}^{\text {diffracted }}(\mathbf{r})= & \mathbf{E}_{\text {inc }}(\mathbf{r})+\frac{i E_{0}}{k r} e^{i k r}\left(\hat{u}_{x}-\theta \cos \phi \hat{u}_{z}\right) \\
& \times \int_{0}^{k a} u d u J_{0}(u \theta) e^{i u^{2} / 2 k r} e^{-u^{2} /(k w)^{2}} .
\end{aligned}
$$

In the limit $z>k w^{2} / 2$ when the incident beam has begun to spread, Eqs. (9) and (41) may be combined to give

$$
\begin{aligned}
\mathbf{E}_{\text {total }}^{\text {diffracted }}(\mathbf{r})= & -i E_{0}\left(\hat{u}_{x}-\theta \cos \phi \hat{u}_{z}\right)\left\{\frac{(k w)^{2}}{2 k z} e^{i k z}\right. \\
& \times \exp \left[\frac{i k\left(x^{2}+y^{2}\right)}{2 z}\left(1-\frac{i k w^{2}}{2 z}\right)^{-1}\right] \\
& \left.-\frac{e^{i k r}}{k r} \int_{0}^{k a} u d u J_{0}(u \theta) e^{i u^{2} / 2 k r} e^{-u^{2} /(k w)^{2}}\right\} .
\end{aligned}
$$

The terms in the curly brackets of Eq. (42) are recognized as the incident field of Eq. (9) minus the Fresnel diffraction field of a circular aperture of radius $a$ which is complementary to the spherical obstacle under consideration. This completes the derivation of the Kirchhoff diffraction integral for a spherical obstacle. ${ }^{43}$ Further, it illustrates what portion of the scattering by a sphere the Kirchhoff diffraction integral corresponds to. It also resolves a paradox concerning the calculation of diffraction, namely when calculating diffraction by a circular obstacle using the Kirchhoff integral, one integrates the radial coordinate as in Eq. (2) from the edge of the obstacle out to infinity, whereas when one calculates diffraction by a spherical obstacle using Mie theory, one sums the partial waves from 1 up to $k a$. The paradox is resolved when the Mie scattered field is added to the incident field. This is equivalent to using the Babinet principle to produce a radial Kirchhoff diffraction integral evaluated from the edge of the obstacle out to infinity.

In Eq. (42), the incident field and the diffracted field of the obstacle's complementary aperture approach their farzone Fraunhofer limits at different distances from the particle. The incident field goes over asymptotically to $\mathbf{E q}$. (10) when $z \gg \theta^{2} k^{3} w^{4}$ and the Fraunhofer diffraction field of the obstacle's complementary aperture is approached when $r \gg k a^{2}$. We now examine the case when the viewing screen is far enough from the particle so that the Fraunhofer limits of both the incident beam and the complementary aperture are applicable and

$$
\begin{aligned}
\mathbf{E}_{\text {total }}^{\text {difracted }}(\mathbf{r})= & \frac{-i E_{0}}{k r} e^{i k r}\left(\hat{u}_{x}-\theta \cos \phi \hat{u}_{z}\right)\left[\frac{(k w)^{2}}{2} e^{-(k w \theta / 2)^{2}}\right. \\
& \left.-\int_{0}^{k a} u d u J_{0}(u \theta) e^{-u^{2} /(k w)^{2}}\right] \\
= & \frac{-i E_{0}}{k r} e^{i k r}\left(\hat{u}_{x}-\theta \cos \phi \hat{u}_{z}\right) \\
& \times \int_{k a}^{\infty} u d u J_{0}(u \theta) e^{-u^{2} /(k w)^{2}}
\end{aligned}
$$

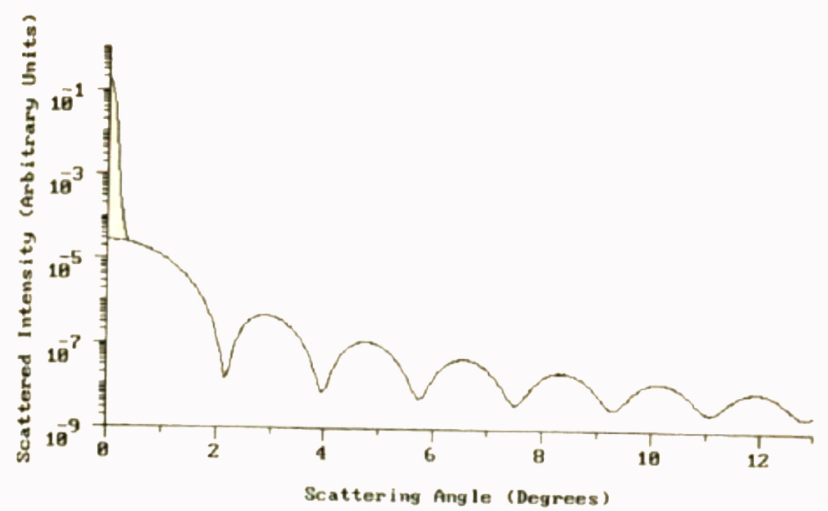

Fig. 5. Diffracted intensity incorrectly calculated without including the incident field (dotted curve) and correctly calculated including the incident field (solid curve) for a Gaussian beam of $\lambda=0.6328 \mu \mathrm{m}$ and $w=100.0 \mu \mathrm{m}$ incident on a spherical water droplet of $n=1.333$ and $a=10.0 \mu \mathrm{m}$. The intensity is calculated at a distance $r=0.1 \mathrm{~m}$ from the droplet in the far zone of both the incident beam and the complementary aperture

The observability of the diffraction pattern of the obstacle's complementary aperture, or the near-forward direction scattered intensity of Eq. (16), is very different depending on whether the width of the incident Gaussian beam satisfies $w \gg a$ or whether $w \lesssim a$. When the focal waist of the Gaussian beam is much wider than the size of the particle, the Gaussian in the integrand of Eq. (43) may be approximated by unity giving

$$
\begin{aligned}
\mathbf{E}_{\text {total }}^{\text {diffracted }}(\mathbf{r})= & \frac{-i E_{0}}{k r} e^{i k r}\left(\hat{u}_{x}-\theta \cos \phi \hat{u}_{z}\right) \\
& \times\left(\frac{(k w)^{2}}{2} e^{-(k w \theta / 2)^{2}}-(k a)^{2} \frac{J_{1}(k a \theta)}{k a \theta}\right) .
\end{aligned}
$$

The square of this electric field is graphed in Fig. 5. The weak Fraunhofer diffraction pattern of the spherical obstacle extends beyond the far-zone angular spreading of the much stronger incident beam and is thus readily observable. This is the situation encountered in the laboratory observation of diffraction by a single particle described in section 1.

When $w \lesssim a$, the diffracted field of the obstacle's complementary aperture becomes almost equal to the far-zone spreading of the incident beam, and is largely canceled by it when the two expressions are combined in Eqs. (13) and (43)..$^{24,44-45}$ This near cancellation causes the complementary aperture diffraction pattern to be unobservable for $w \lesssim a$. The actual diffraction pattern for this situation is the residual amount left after the near-cancellation. This is given by the second equality of Eq. (43). It physically corresponds to the Gaussian tail of the incident beam interacting with the edges of the particle and is illustrated in Fig. 6.

These results have an impact on how diffraction of a Gaussian beam by an obstacle should be calculated using either Eqs. (13) and (16) or Eq. (42). For plane-wave incidence and far-zone Fraunhofer diffraction by an obstacle, the complementary aperture field or the scattered field 


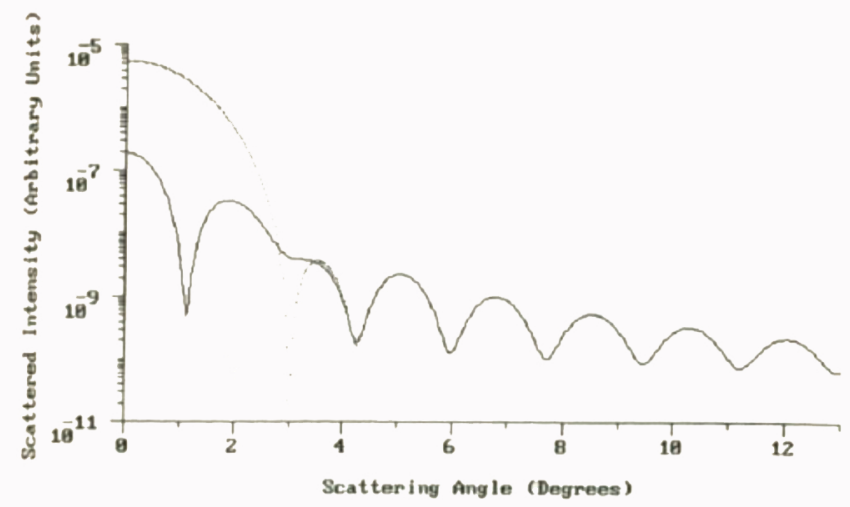

Fig. 6. Diffracted intensity incorrectly calculated without including the incident field (dotted curve) and correctly calculated including the incident field (solid curve) for a Gaussian beam of $\lambda=0.6328 \mu \mathrm{m}$ and $w=7.5$ $\mu \mathrm{m}$ incident on a spherical water droplet of $n=1.333$ and $a=10.0 \mu \mathrm{m}$. The intensity is calculated at a distance $r=0.1 \mathrm{~m}$ from the droplet in the far zone of both the incident beam and the complementary aperture.

is in practice never subtracted from or added to the incident field, and one often sees the statement that the Fraunhofer diffraction pattern of an obstacle and its complementary aperture are identical. ${ }^{46,47}$ Gaussian beam theory with $w \gg a$ gives a justification of this neglect of the incident field. On the other hand, for Gaussian beam incidence with $w \lesssim a$, the neglect of the incident field in far zone is incorrect since diffraction does not reside solely in the diffracted field of the obstacle's complementary aperture. It also resides in the interference between the complementary aperture field and the incident field. For this situation, to throw away the incident field is to throw away part of the answer to the diffraction problem.

These results also serve to refine our intuitive ideas concerning diffraction. One often thinks of diffraction as the bending of light rays that pass the sphere with impact parameters sufficiently large so as to miss striking it. ${ }^{48,49}$ This picture more appropriately describes the tunneling reflection of partial waves with $l>k a$. A better picture of diffraction of light rays by an obstacle is an interaction of the light with the edges of the obstacle.

\section{ACKNOWLEDGMENT}

This work was supported by the National Aeronautics and Space Administration Grant No. NCC 3-204.

'M. Born and E. Wolf, Principles of Optics, Sixth Edition (Pergamon, New York, 1980), Sec. 11.5.

${ }^{2}$ Ref. 1, Sec. 11.8.

${ }^{3}$ E. Hecht, Optics, Second Edition (Addison-Wesley, Reading, MA, 1987), Chap. 10.4

${ }^{4} \mathrm{~J}$. W. Goodman, Introduction to Fourier Optics (McGraw-Hill, New York, 1968), Chap. 3.

D. S. Burch, "Fresnel diffraction by a circular aperture," Am. J. Phys. 53, 255-260 (1985).

${ }^{6}$ J. J. Bowman, T. B. A. Senior, and P. L. E. Uslenghi, Electromagnetic and Acoustic Scattering by Simple Shapes (Hemisphere, New York, 1987), Chap. 14.
'H. C. van de Hulst, Light Scattering by Small Particles (Dover, Ne' York, 1981), Chap. 9.

${ }^{8}$ Reference 6, Chap. 4.

${ }^{9}$ Reference 7, Chap. 15.

${ }^{10}$ Reference 3, Sec. 10.3.10-10.3.11.

"L. Brillouin, "The scattering cross section of spheres for electromag netic waves," J. Appl. Phys. 20, 1110-1125 (1949).

${ }^{12} \mathrm{P}$. M. Rinard, "Large-scale diffraction patterns from circular objects, Am. J. Phys. 44, 70-76 (1976)

${ }^{13}$ E. A. Hovenac, "Fresnel diffraction by spherical obstacles," Am. J Phys. 57, 79-84 (1989).

${ }^{14}$ G. E. Sommargren and H. J. Weaver, "Diffraction of light by as opaque sphere. I: Description and properties of the diffraction pattern,' Appl. Opt. 29, 4646-4657 (1990)

${ }^{15} \mathrm{G}$. Gouesbet, B. Maheu, and G. Grehan, "Light scattering from : sphere arbitrarily located in a Gaussian beam, using a Bromwich for malism," J. Opt. Soc. Am. A5, 1427-1443 (1988).

${ }^{16}$ J. P. Barton, D. R. Alexander, and S. A. Schaub, "Internal and near surface electromagnetic fields for a spherical particle irradiated by a focused laser beam," J. Appl. Phys. 64, 1632-1639 (1988).

${ }^{17}$ Reference 3, p. 397.

${ }^{18}$ Reference 3, Chap. 10.5

${ }^{19}$ Reference 4, Chap. 3.6.

${ }^{20}$ S. Ganci, "An experiment on the physical reality of edge-diffracted waves," Am. J. Phys. 57, 370-373 (1989).

${ }^{21}$ L. W. Davis, "Theory of electromagnetic beams," Phys. Rev. A 19 1177-1179 (1979)

${ }^{22} \mathrm{~A}$. Wunsche, "Transition from the paraxial approximation to exact solutions of the wave equation and application to Gaussian beams," J Opt. Soc. Am. A 9, 765-774 (1992).

${ }^{23}$ F. Slimani, G. Grehan, G. Gouesbet, and D. Allano, "Near-field Lorenz-Mie theory and its application to microholography," Appl. Opt. 23, 4140-4148 (1984)

${ }^{24}$ F. Guilloteau, G. Grehan, and G. Gouesbet, "Optical levitation experiments to assess the validity of the generalized Lorenz-Mie theory," Appl. Opt. 31, 2942-2951 (1992).

${ }^{25}$ W. J. Wiscombe, "Improved Mie scattering algorithms," Appl. Opt. 19, 1505-1509 (1980).

${ }^{26} \mathrm{H}$. M. Nussenzveig, "High-frequency scattering by a transparent sphere. I. Direct reflection and transmission," J. Math. Phys. 10, 82124 (1969).

${ }^{27}$ J. A. Lock, "Cooperative effects among partial waves in Mie scattering," J. Opt. Soc. Am. A5, 2032-2044 (1988).

${ }^{28} \mathrm{~J}$. A. Lock, "The contribution of high-order rainbows to the scattering of a Gaussian laser beam by a spherical particle," J. Opt. Soc. Am. A 10, 693-706 (1993)

${ }^{29}$ Ref. 7 , Sec. 12.31 .

${ }^{30}$ L. G. Guimaraes and H. M. Nussenzveig, "Theory of Mie resonances and ripple fluctuations," Opt. Commun. 89, 363-369 (1992).

${ }^{31}$ H. M. Nussenzveig, "High-frequency scattering by a transparent sphere. II. Theory of the rainbow and the glory," J. Math. Phys. 10, 125-176 (1969)

${ }^{32} \mathrm{M}$. V. Berry, "Semi-classical scattering phase shifts in the presence of metastable states," Proc. Phys. Soc. (London) 88, 285-292 (1966).

${ }^{33}$ Ref. 7, Sec. 12.32 .

${ }^{34}$ M. V. Berry, "Uniform approximations for glory scattering and diffraction peaks," J. Phys. B2, 381-392 (1969).

${ }^{35}$ H. M. Nussenzveig and W. J. Wiscombe, "Diffraction as tunneling," Phys. Rev. Lett. 59, 1667-1670 (1987).

${ }^{36} \mathrm{H}$. M. Nussenzveig, "Uniform approximation in scattering by spheres," J. Phys. A21, 81-109 (1988)

${ }^{37}$ H. M. Nussenzveig, "Tunneling effects in diffractive scattering and resonance," Comments At. Mol. Phys. 23, 175-187 (1989).

${ }^{38} \mathrm{H}$. M. Nussenzveig and W. J. Wiscombe, "Complex angular momentum approximation to hard-core scattering," Phys. Rev. A 43, 20932112 (1991)

${ }^{39} \mathrm{H}$. M. Nussenzveig, "High-frequency scattering by an impenetrable sphere," Ann. Phys. (N.Y.) 34, 23-95 (1965).

${ }^{40} \mathrm{G}$. Grehan, B. Maheu, and G. Gouesbet, "Scattering of laser beams by Mie scatter centers: Numerical results using a localized approximation," Appl. Opt. 25, 3539-3548 (1986).

${ }^{41}$ B. Maheu, G. Grehan, and G. Gouesbet, "Ray localization in Gaussian beams," Opt. Commun. 70, 259-262 (1989).

${ }^{42}$ G. Arfken, Mathematical Methods for Physicists, 3rd ed. (Academic, New York, 1985), Eq. (11.152). 
${ }^{43}$ Different derivations of this result for plane wave incidence are carried out in Refs. 35 and 38.

${ }^{44}$ J. P. Campbell and L. G. De Shazer, "Near-fields of truncatedGaussian apertures," J. Opt. Soc. Am. 59, 1427-1429 (1969).

${ }^{45}$ P. M. Morse and H. Feshbach, Methods for Theoretical Physicists (McGraw-Hill, New York, 1953), pp. 1551-1555.

${ }^{46}$ Reference 3, Sec. 10.3.11 and Fig. 10.78.
${ }^{47}$ J. D. Jackson, Classical Electrodynamics (Wiley, New York, 1962), Sec. 9.7.

${ }^{48}$ R. M. Eisberg. Fundamentals of Modern Physics (Wiley, New York, 1961), pp. 458-459.

${ }^{49}$ C. F. Bohren and D. R. Huffman, Absorption and Scattering of Light by Small Particles (Wiley, New York, 1983), p. 110.

Post-print standardized by MSL Academic Endeavors, the imprint of the Michael Schwartz Library at Cleveland State University, 2017. 